\title{
ANÁLISIS DE REDUCCIÓN DE RUIDO EN SEÑALES EEG ORIENTADO AL RECONOCIMIENTO DE PATRONES
}

\author{
Cristian Guarnizo Lemus ${ }^{1}$
}

\section{Resumen}

En este artículo se presenta un estudio sobre la reducción de ruido de fondo (electrónico) en señales electroencefalográficas (EEG) utilizando la transformada wavelet, asumiendo que las características extraídas son susceptibles al ruido inherente en la señal y común entre clases. Adicionalmente se observa la incidencia en la separación de las muestras en el espacio de características a partir de un clasificador bayesiano lineal. Se obtiene un incremento del $1 \%$ en la media del porcentaje de acierto, al realizar la reducción de ruido en la identificación de dos estados funcionales.

\section{Palabras clave}

Wavelet, EEG, reducción de ruido.

\section{Alostract}

A study on background noise reduction (denoising) on EEG signals using wavelet transform is presented, assuming that extracted features are susceptible to common noise within classes; besides, the feature space separability is compared using a linear Bayesian classifier. An increment of $1 \%$ in the average recognition

1 Ingeniero Electricista. Universidad Tecnológica de Pereira, Maestría en Ingeniería Eléctrica. Docente Ocasional ITM Institución Universitaria. 
[68]

rate is reached performing noise reduction in the identification of two functional states.

\section{Key words}

Wavelet, EEG, denoising. 


\section{INTRODUCCIÓN}

En todo proceso de adquisición de señales biológicas, las mediciones tomadas poseen cierto grado de distorsión o se alteran debido a varios factores, como, ruido térmico, interferencia electromagnética o movimientos del paciente (Donoho \& Johnstone, 1992).

Debido a que el ruido es un factor común en las señales adquiridas hace que las muestras de diferentes clases posean cierta similitud en su comportamiento estadístico, por esta razón en general en los procesos de reconocimiento de patrones se utiliza una etapa de preprocesamiento.

La mayoría de los métodos actuales para la reducción de ruido en bioseñales se basan en la transformada wavelet, debido que fácilmente puede representar (realzar) los eventos fisiológicos de interés en el dominio tiempo-escala, además que son adecuadas para procesar señales de naturaleza no estacionaria. En (Messer, Agzarian, \& Abbott, 2001) se presenta un análisis de reducción de ruido en señales fonocardiográficas variando cada parámetro involucrado en el preprocesamiento (onda wavelet, tipo de umbralización, valor del umbral y escalamiento del umbral), se analiza la relación señal a ruido de las señales procesadas. El mayor problema en este método es que se debe agregar ruido a una señal que inherentemente contiene perturbaciones de otras fuentes, solo para verificar que el método reduce ese ruido agregado. Un trabajo similar al anterior pero aplicado sobre señales EEG es propuesto en (Prasad, Siddaiah, \& Rao, 2008).

Los trabajos de reducción de ruido en EEG son muy específicos, en los cuales se trata de eliminar artefactos visibles en la señal, como la perturbación generada por el movimiento de los ojos (Senthil, Arumuganathan, Sivakumar, \& Vimal, 2008) (Krishnaveni, Jayaraman, Aravind, Hariharasudhan, \& Ramadoss, 2006).

En las señales EEG se han propuesto diferentes métodos de caracterización basados en la transformada wavelet para la posterior identificación de los estados funcionales (Subasi, 2007) (Ting, 
Guo-zheng, Bang-hua, \& Hong, 2008) (Jahankhani, Kodogiannis, \& Revett, 2006), pero no se hace un estudio de la reducción del ruido y como este afecta el análisis futuro de las señales.

En (Jahankhani, Kodogiannis, \& Revett, 2006) se obtiene un porcentaje de acierto de $97 \%$ en la clasificación de dos estados funcionales utilizando la combinación de 16 clasificadores basados en redes neuronales. De igual manera en (Subasi, 2007) se obtiene un porcentaje de acierto de $94.5 \%$ utilizando mezclas de expertos. En los trabajos anteriores se obtuvieron más muestras de la base de datos original al segmentar los registros originales, de esa forma se obtiene un conjunto de datos formado por 1000 muestras para entrenamiento y 600 para la validación en la identificación de dos estados funcionales.

\section{Materiales y métodos}

En la reducción de ruido generalmente se observa la relación señal a ruido (SNR) de la señal resultante, como medida de desempeño del método de preprocesamiento. En las tareas de reconocimiento de patrones se busca obtener un espacio de características en el que las clases estén claramente separadas, esta última depende de la calidad de las señales y del método de caracterización. Por esta razón se pretende obtener una mejor separación entre clases reduciendo la componente de ruido común en todas las señales.

\subsection{Datos de entrenamiento}

La base de datos utilizada en este experimento está disponible públicamente, y es descrita en (Andrzejak, 2001). La base de datos completa está compuesta por 5 conjuntos (A-E) donde cada uno contiene 100 segmentos de EEG de un solo canal. Los conjuntos A y B están compuestos de señales superficiales de EEG tomadas de voluntarios saludables. Ambos conjuntos tienen grabaciones de los voluntarios en estado despierto, pero en el conjunto A tienen los ojos abiertos. Los conjuntos C, D y E son grabaciones de 5 
pacientes que posteriormente se les diagnosticó quirúrgicamente tener zona epileptógena. Todas las señales EEG fueron registradas con un sistema de amplificación de 128 canales, los datos fueron digitalizados con una resolución de 12 bits y muestreados a 173.61 Hz. En este trabajo se utiliza los conjuntos de datos A y E como en (Subasi, 2007).

\subsection{Reducción de ruido con wavelets}

La reducción de ruido de fondo trata de disminuir el ruido inherente en la adquisición de la señal. Se pretende reducir el ruido de fondo empleando técnicas basadas en la transformada wavelet discreta (DWT), las cuales han mostrado una estimación mejor de la señal sin ruido y posteriormente observar si el acierto en la identificación de las diferentes clases se incrementa o disminuye.

En la reducción de ruido de fondo se busca encontrar una aproximación cercana a los valores $x_{n}$ de $y_{n}$ dado el siguiente modelo:

$$
y_{n}=x_{n}+\eta_{n} \quad n=1, \ldots, N
$$

donde $x$ es la señal sin perturbaciones, $\eta$ es un proceso aleatorio blanco Gaussiano con media $\mu=0, \mathrm{~N}$ es la longitud de la señal.

La transformada wavelet tiene propiedades (momentos de desvanecimiento) en sus funciones base que hacen posible obtener una muy buena aproximación de señales que contienen ruido aditivo.

Si las funciones base de la DWT son ortonormales, entonces la transformada del ruido blanco Gaussiano $\eta_{n}$ corresponde a otro proceso de estructura blanca Gaussiana $\varepsilon_{n}$, de la misma amplitud de $\eta_{n}$ (Jansen, 2001), entonces

$$
W\left\{y_{n}\right\}=W\left\{x_{n}\right\}+W\left\{\eta_{n}\right\}
$$

$\mathrm{Al}$ resolver (0.2) para $x_{n}$, se obtiene 
En general, el valor de $\varepsilon_{n}$ no se conoce, así que a la componente del ruido se le asigna un valor $\lambda$, tal que

$$
x_{n} \approx W^{-1}\left\{W\left\{y_{n}\right\}-\lambda\right\}
$$

La remoción del ruido mediante la técnica de reducción de ruido (denoising), consiste en eliminar la contribución correspondiente al valor estimado $\lambda$, de cada uno de los coeficientes wavelet en $W\left\{y_{n}\right\}$. La primera forma consiste en emplear el procedimiento de limitación por nivel o umbralización, la cual puede estar motivada por las siguientes razones (Jansen, 2001):

- La propiedad de no correlación de la DWT genera una señal poco densa; la mayoría de los coeficientes libres de ruido son cero o cercanos a cero.

- El ruido está distribuido de igual forma en todos los coeficientes.

- Se supone que la potencia del ruido es mucho menor que la de la señal útil, de modo que se puede distinguir, para la señal útil, el aporte de los coeficientes wavelet.

El valor de umbral corresponde a un compromiso entre la cercanía del ajuste y el suavizado: un valor pequeño da como resultado una señal de salida con forma cercana a la de la entrada, de tal manera, que la remoción de ruido es insuficiente. Por el contrario, un valor de umbral muy alto produce una señal de salida con muchos coeficientes wavelet iguales a cero; esto es, con demasiada distorsión y pérdida de la información (Jansen, 2001).

\subsection{Funciones de umbralización}

Los valores de los coeficientes son modificados a partir del valor del umbral, empleando unas funciones de limitación, las propuestas en (Johnstone, 1992) se presentan a continuación:

Umbral fijo: Los coeficientes wavelet más pequeños están dominados por el ruido, mientras que los coeficientes con valores 
absolutos grandes poseen más información de la señal que del ruido. Por esto, los coeficientes más pequeños según el valor absoluto y que están por debajo del umbral fijo $\lambda$, se reemplazan por cero, de la siguiente manera:

$$
\hat{d}_{k}^{l}= \begin{cases}0, & \left|d_{k}^{l}\right|<\lambda \\ d_{k}^{l}, & \left|d_{k}^{l}\right| \geq \lambda\end{cases}
$$

donde $d_{k}^{l}$ es el coeficiente de detalle wavelet $k$ del nivel $l$. El limitador de umbral fijo se usa cuando se desea obtener el código wavelet, lo más corto posible. Las secuencias largas de ceros que usualmente se obtienen en el vector limitado de la descomposición wavelet, se codifican eficientemente. Aunque esta función de umbralización es buena para reproducir señales con discontinuidades o cambios abruptos, también produce artefactos en la señal reconstruida debido a la discontinuidad presente en el valor del umbral $\lambda$, por este motivo se propone la siguiente función de umbralización.

Umbral flexible: En este caso, se reducen todos los coeficientes hacia el origen, de la siguiente forma:

$$
\hat{d}_{k}^{l}= \begin{cases}0, & \left|d_{k}^{l}\right|<\lambda \\ \operatorname{sgn}\left(d_{k}^{l}\right)\left(d_{k}^{l} \mid-\lambda\right), & \left|d_{k}^{l}\right| \geq \lambda\end{cases}
$$

donde sgn es la función signo. El problema de esta función es que la señal reconstruida presentará una diferencia menor en amplitud a la señal original.

\subsection{Valor del umbral}

Para determinar el valor del umbral en general se emplean 4 estimadores estadísticos que buscan un umbral para el cual los valores menores a éste pertenezcan al ruido.

Universal: El valor del umbral depende de la cantidad de muestras de la señal:

$$
\lambda=\sqrt{2 \log (N)}
$$


Estimador de riesgo no sesgado de Stein (SURE): El valor del umbral se encuentra minimizando una función de riesgo

$$
\lambda=\sqrt{N V\left(k_{\min }\right)}
$$

Donde $N V(k)$ es un vector de datos (coeficientes) al cuadrado, organizado en orden ascendente. El riesgo en el índice $k$ está dado por:

$$
R(k)=\frac{N-2 k+\sum_{j=1}^{k} N V(j)+(N-k) N V(N-k)}{n}
$$

Se selecciona para calcular el valor del umbral el índice $k_{\min }$ que minimice el riesgo del vector de riegos $R(k)$.

SURE Heurístico: Se selecciona el menor valor de los umbrales estimados por los dos métodos anteriores. Este umbral es el más conservativo con respecto a la señal, retiene más ruido.

Minimax: Se basa en la regla de Minimax, definida para una serie de datos como:

$$
\lambda=0.3936+0.1829(\log (N) / \log (2))
$$

\subsection{Escalamiento}

El valor del umbral se puede ponderar de acuerdo con los valores de los coeficientes por escala, de esta forma el umbral puede ser dependiente del nivel de ruido encontrado por nivel. Existen tres formas en las cuales se puede ponderar el valor del umbral determinado por alguno de los estimadores anteriores:

Global (one): A todos los coeficientes de todos los niveles se les aplica el valor del umbral sin ponderar.

Ponderación primer nivel $(\operatorname{sln})$ : Se calcula el nivel de ruido de los coeficientes de detalle del primer nivel de descomposición a partir de la siguiente ecuación:

$$
\hat{\sigma}=\frac{\text { mediana }\left(\left|d_{k}^{1}\right|\right)}{0.6745}
$$


Donde $\hat{\sigma}$ es el nivel del ruido estimado, el valor del umbral se pondera entonces:

$$
\lambda^{*}=\hat{\sigma} \lambda
$$

Ponderación por nivel $(\mathrm{mln})$ : En este caso el valor del umbral se pondera por escala a partir de la estimación del nivel de ruido en la escala.

Un esquema para la reducción de ruido empleando la transformada wavelet se puede observar en la figura 1.

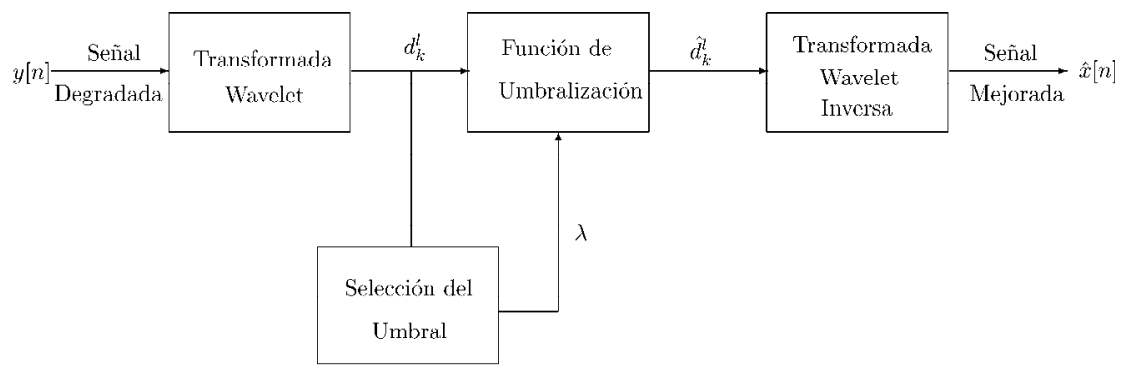

FIgURA 1. ESQUEMA DE REDUCCIÓN DE RUIDO

\subsection{Caracterización}

La caracterización se realiza calculando la transformada wavelet con tres niveles de descomposición, a cada muestra transformada en el espacio wavelet, se le calculan los siguientes parámetros o medidas por nivel de descomposición (XU, 2004):

$$
\begin{aligned}
& \mu_{n}^{l}=\frac{1}{K} \sum_{k=1}^{K}\left|d_{k}^{l}\right| \\
& E^{l}=\frac{1}{2} \sum_{k=1}^{K}\left(d_{k}^{l}\right)^{2} \\
& m_{n}^{l}=\max \left(\left|d_{k}^{l}\right|\right) \\
& S V D=\sqrt{\Delta\left(C^{T} C\right)}
\end{aligned}
$$


Donde $\mu_{n}^{l}$ es la media normalizada, $E^{l}$ es la energía y $m_{n}^{l}$ es el máximo absoluto de los coeficientes de la escala $l, S V D$ es la raíz cuadrada de los valores propios de la matriz $C^{T} C$. La matriz $C$ se obtiene indexando en filas los coeficientes de los diferentes niveles de descomposición como se muestra en la figura 2 .

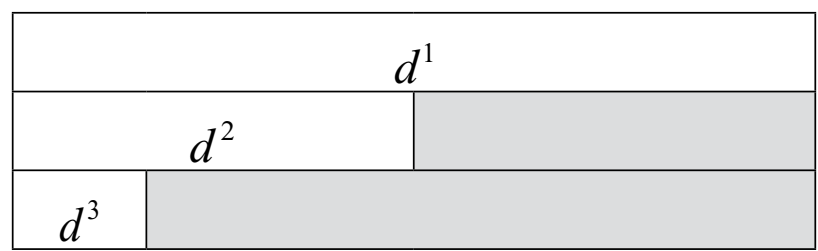

Figura 2. CONSTRUCCIÓN DE LA MATRIZ C, REGIÓN GRIS COMPUESTA DE CEROS

\section{Resultados y Discusión}

Primero se selecciona la base wavelet para caracterizar las señales EEG, debido a que se pretende observar cómo cambia el espacio de características al reducir el ruido en las señales, se selecciona la base que entregue el mejor porcentaje de acierto. Los valores de clasificación de las características se obtienen utilizando un clasificador bayesiano lineal con validación cruzada de cinco particiones. Los mejores porcentajes por familia wavelet se pueden observar en la tabla 1.

Tabla 1. Porcentaje de acierto para diferentes bases waVelet

\begin{tabular}{|c|c|c|c|}
\hline Base DWT & A & E & Total \\
\hline Db1 & $100.00 \pm 0.00$ & $90.00 \pm 7.91$ & $95.00 \pm 3.95$ \\
\hline Coif1 & $100.00 \pm 0.00$ & $84.00 \pm 5.48$ & $92.00 \pm 2.74$ \\
\hline Sym10 & $100.00 \pm 0.00$ & $86.00 \pm 4.18$ & $93.00 \pm 2.09$ \\
\hline
\end{tabular}

Posteriormente se realiza el denoising de todas las señales y se obtienen los porcentajes de acierto para cada caso variando la base wavelet (Coiflet 1-5, Daubechies 1-10 y Symlet 2-10), la función de 
umbralización, el valor del umbral y el escalamiento del umbral (576 pruebas diferentes).

En la figura 3 se puede observar la variación del porcentaje de acierto al variar la base, función de umbralización, umbral y escalamiento, respectivamente.

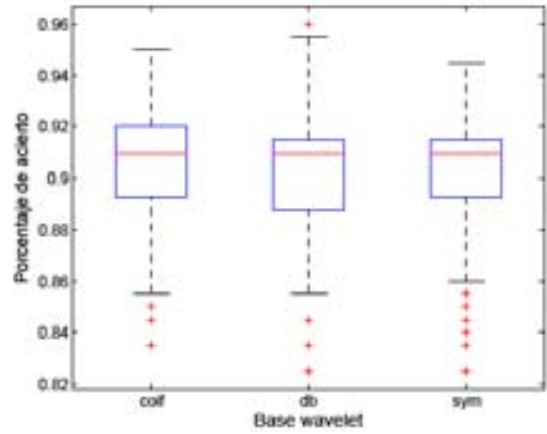

(a)

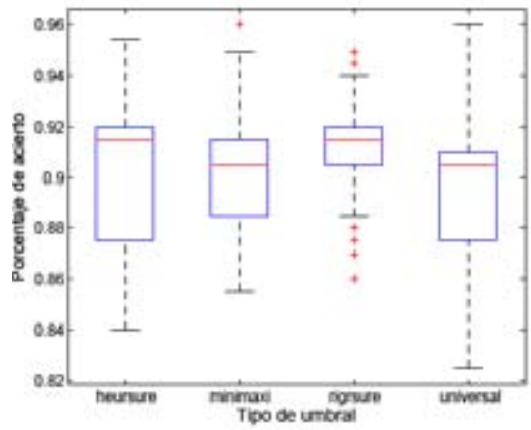

(c)

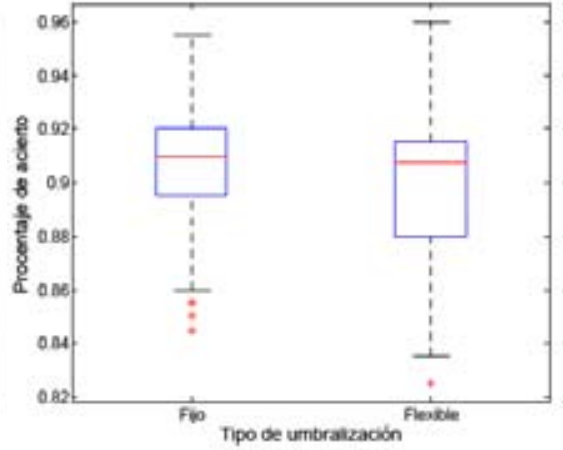

(b)

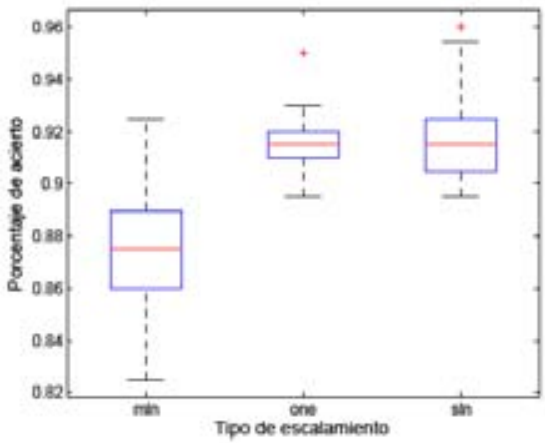

(d)

Figura 3. Boxplot de cada parámetro en la Reducción de Ruido. (a) Base wavelet.

(B) Función de umbralización. (c) UMBral. (D) Escalamiento

De la figura 3, se observa que el cambio de la base no influye en el comportamiento del clasificador. El tipo de umbralización flexible presenta menores porcentajes de acierto que la fija. El parámetro 
que influye en el porcentaje de acierto al reducir el ruido en las señales es el tipo de escalamiento, si el escalamiento es global o ponderado por el primer nivel se obtienen porcentajes más altos. En la tabla 2 se muestran los porcentajes de acierto que superarán en la media a aquel entregado sin realizar un preprocesamiento sobre la señal.

Tabla 2. Porcentaje de acierto con mayor tendencia Al filtrar

\begin{tabular}{|c|c|c|c|}
\hline MÉтоDо & A & E & TотAL \\
\hline $\begin{array}{c}\text { Db1, HSURE, fijo, } \\
\text { sln. }\end{array}$ & $100.00 \pm 0.00$ & $92.00 \pm 2.74$ & $96.00 \pm 1.37$ \\
\hline $\begin{array}{c}\text { Db1, minimax, } \\
\text { flexible, sln. }\end{array}$ & $100.00 \pm 0.00$ & $92.00 \pm 5.70$ & $96.00 \pm 2.85$ \\
\hline $\begin{array}{c}\text { Db1, universal, } \\
\text { flexible, sln. }\end{array}$ & $100.00 \pm 0.00$ & $91.00 \pm 7.42$ & $95.50 \pm 3.71$ \\
\hline
\end{tabular}

A partir de la tabla anterior se observa que al realizar la reducción de ruido se obtiene una mejora del 1\% en la tendencia de central de los porcentajes de acierto. Las características extraídas son lo suficientemente robustas para la representación de las señales EEG de la base de datos utilizada debido que no se ven influenciadas por el ruido inherente en las señales, esto se refleja en los resultados obtenidos al realizar o no el filtrado sobre las señales. En comparación con el trabajo de (Subasi, 2007) donde se obtiene un porcentaje de acierto de $94.5 \%$ utilizando mezclas de expertos, en este trabajo se presenta un mayor porcentaje de acierto utilizando un clasificador más sencillo. 


\section{Conclusiones}

En la reducción de ruido utilizando la transformada wavelet, el parámetro que influye en el porcentaje de acierto es el tipo de escalamiento utilizado.

No es necesario realizar la reducción de ruido empleando el método de caracterización propuesto, debido a que los resultados presentados con ruido y sin reducción de éste no presentan una diferencia significativa.

\section{Biblografía}

Andrzejak, R. G. (2001). Indications of nonlinear deterministic and finite dimensional structures in time series of brain electrical activity: Dependence on recording region and brain state. PHYSICAL REVIEW E.

Donoho, D., \& Johnstone, I. (1992). Ideal spatial adaptation by wavelet shrinkage. Biometrika, 425-455.

Jahankhani, P., Kodogiannis, V., \& Revett, K. (2006). EEG Signal Classification Using Wavelet Feature Extraction and Neural Networks. IEEE John Vincent Atanasoff 2006 International Symposium on Modern Computing.

Jansen, M. (2001). Lecture notes in Statistics: Noise reduction by wavelet thresholding. Springer Verlag.

Krishnaveni, V., Jayaraman, S., Aravind, S., Hariharasudhan, V., \& Ramadoss, K. (2006). Automatic Identification and Removal of Ocular Artifacts from EEG using Wavelet Transform. MEASUREMENT SCIENCE REVIEW, 45-57.

Messer, S. R., Agzarian, J., \& Abbott, D. (2001). Optimal wavelet denoising for phonocardiograms. Microelectronics journal, 931-941.

Prasad, V., Siddaiah, P., \& Rao, P. (2008). Denoising of Biological Signals Using Different Wavelet Based Methods and Their Comparison. Asian Journal of Information Technology, 146-149.

Senthil, P., Arumuganathan, R., Sivakumar, K., \& Vimal, C. (2008). A Wavelet based Statistical Method for De-Noising of Ocular Artifacts in EEG Signals. IJCSNS International Journal of Computer Science and Network Security, 87-92. 
Subasi, A. (2007). EEG signal classification using wavelet feature extraction and a mixture of expert model. Expert Systems with Applications, 1084-1093.

Ting, W., Guo-zheng, Y., Bang-hua, Y., \& Hong, S. (2007). EEG feature extraction based on wavelet packet decomposition for brain computer interface. Measurement, 618-625.

Zhang, X., Yang, Y., \& Xu, X. (2004). Clinical detection and movement recognition of neuro signals. Journal of Zheijang University SCIENCE. 